\title{
ORIGINAL
}

\section{ESTUDIO MICROBIOLÓGICO DE LAS COMIDAS SERVIDAS EN LOS COMEDORES ESCOLARES DE LA ISLA DE TENERIFE}

Julia Campos Díaz, Cristobalina Rodríguez Alvarez, Antonio Sierra López, Ángeles Arias Rodríguez

Área de Medicina Preventiva y Salud Pública. Universidad de La Laguna. Santa Cruz de Tenerife.

RESUMEN

Fundamento: Valorar la calidad higienico-sanitaria de las com-as servidas en comedores escolares con la finalidad de conocer si es adecuada o por el contrario su ingesta puede representar un grave problema de salud para este colectivo de alto riesg

Métodos: Estudio epidemiológico descriptivo transversal. Se alizan 898 muestras de alimentos recogidos en comedores de 10 colegios de Tenerife, seleccionados por un muestreo probabilistico aleatorio estratificado, 58 con elaboración propia de los alimento (gestión directa) y 43 con comidas servidas por un catering (elaboración contratada).

Resultados: En ninguna de las muestras analizadas se aislaron os patógenos Salmonella spp. y Listeria monocytogenes. El 79\% de los alimentos estudiados pesentó recuentos para este puśn $(91 \%)$ de ensaladas y $(85 \%)$ de segundos platos. Para Enterobacteriaceae totales, el $15 \%$ de las muestras analizadas fueron positivas. Escherichia coli se aisló en el 24\% de las ensaladas, el $4 \%$ de los complementos y el $1 \%$ de los segundos platos y Staphylococcus aureus se aislo en tres alimentos. Los recuentos más elevados se obtuvieron para los microorganismos aerobios mesófilos totales. De total de muestras a alizadas un $8,24 \%$ de las nismas superaron uno

Conclusiones: La calidad microbiológica de las comidas servidas en los comedores escolares es aceptable, si bien al existir un porcentaje de alimentos que superan los limites establecidos en microorganismos indicadores y testigos de falta de higiene y al ser lo escolares un colectivo de alto riesgo, será necesaria la revisión de la vigilancia en los puntos de control crítico.

Palabras clave: Alimentación escolar, Higiene alimentaria. Servicios de Salud escolar. Salud pública.

Correspondenci

Angeles Arias Rodríguez.

Área de Medicina Preventiva y Salud Pública. Facultad de Medicina. Campus de Ofra 38071. La Laguna. Tenerife. Islas Canarias. España.

Teléfono: 922319380

Fax : 922319378

Correo electrónico: angarias@ull.es

\section{ABSTRACT}

Microbiological Study of the Meals Served in School Lunchrooms on the Island of Tenerife, Spain

Background: School lunchrooms and catered meals are of major importance from the Public Health standpoint. This study is aimed at evaluating the microbiological quality of the meals served in school lunchrooms for the purpose of ascertaining whether it is
suitable or, to the contrary, the intake thereof may involve a serious health problem for this high-risk group.

Methods: A transversal descriptive epidemiological study. An analysis was conducted of a total of 898 food samples collected from he lunchrooms at 101 schools in Tenerife, selected by a stratified prepared at the school proper (direcedure, fifty-eight of which were meals served by a catering firm (prepared under contract).

Results: No disease-causing Salmonella spp. or Listeria monocytogenes bacteria were isolated from any of the samples. A $(91 \%)$ in alds and $(85 \%)$ in min courses. A total $15 \%$ of the samples analyzed tested positive for total Enterobacteriaceee. Escherichia coli was isolated in $24 \%$ of the salads, in $4 \%$ of the side dishes and in $1 \%$ of the main dishes, Staphylococcus aureus having in isolated in three foods. The highest counts were found for the total aerbic mesophyllic microorganisms. A total $8.24 \%$ of the sample analyzed exceeded one or more of the limits stipulated for the parameters studies.

Conclusions: The microbiological quality of the meals served in these school lunchrooms is acceptable, although due to a certain percentage of the foods having exceeded the stipulated limits for microorganisms indicative of and revealing a lack of hygiene, and schoolchildren being a high-risk group, a revision of the surveillance related to critical checkpoints will be necessary.

Key words: School canteen. Food hygiene, School health services. Public health. 


\section{INTRODUCCIÓN}

En la actualidad, un gran porcentaje de la población escolar realiza la comida principal en su centro de enseñanza. Los comedores de centros docentes y la restauración colectiva dirigida a escolares tiene una especial importancia desde el punto de vista de la salud pública, puesto que se trata de comedores sociales utilizados por un grupo de población tipificado como colectivo vulnerable ${ }^{1,2}$.

Los alimentos en ocasiones pueden vehiculizar microorganismos patógenos o sus toxinas, con el consiguiente riesgo para la salud del consumidor, pudiendo causar brotes de origen alimentario, lo que puede representar un grave problema de salud pública. Es imprescindible un control eficaz de la higiene, a fin de evitar las consecuencias perjudiciales que derivan de las enfermedades y los daños provocados por los alimentos y por el deterioro de los mismos para la salud y para la economía. La calidad higiénica de los alimentos es uno de los aspectos que van a influir de forma directa en la salud, ya que su alteración, adulteración o contaminación, tanto química como biológica puede afectar seriamente a la salud $^{3}$.

El control de calidad de un comedor escolar comienza con la supervisión del suministro de alimentos en cantidades físicas o el cuidado de los platos preparados. Una vez el menú está en disposición de ser suministrado es fundamental vigilar sus características organolépticas, su calidad higiénico-sanitaria y los procedimientos empleados en su conservación en el tiempo que transcurre desde su preparación hasta su consumo ${ }^{1,2}$.

El análisis microbiológico de un alimento permite conocer sus fuentes de contaminación, valorar las normas de higiene utilizadas en la elaboración y manipulación de alimentos, detectar la posible presencia de microbiota patógena que suponga un riesgo para la salud del consumidor (siendo éste uno de los objetivos más importantes en microbiología alimentaria) y establecer en qué momento se producen fenómenos de alteración en los distintos alimentos, con el fin de delimitar su período de conservación ${ }^{4}$.

En la actual Reglamentación española, el RD 3484/2000 de 29 de diciembre establece las normas de higiene para la elaboración, distribución y comercio de las comidas preparadas, y clasifica los tipos de comidas que se sirven en los comedores escolares en dos grupos:

- Grupo A: comidas preparadas sin tratamiento térmico y comidas preparadas con tratamiento térmico, que lleven ingredientes no sometidos a tratamiento térmico

- Grupo B: comidas preparadas con tratamiento térmico.

En ambos grupos establece como microorganismos indicadores a los aerobios mesófilos y Enterobacteriaceae lactosa positiva o coliformes y como testigos de falta de higiene a Escherichia coli y Staphylococcus aureus. Además el Real Decreto establece la obligatoriedad del análisis para la detección de los patógenos a Salmonella spp. y Listeria monocytogenes ${ }^{5}$. Los microorganismos indicadores deben ayudar a juzgar el buen funcionamiento del establecimiento y el procedimiento de autocontrol aplicado en la elaboración de las comidas preparadas. Un contenido de microorganismos testigo o de falta de higiene superior al establecido en la normativa vigente implicará la revisión de los métodos de vigilancia aplicados en los puntos de control crítico y, de superarse los límites establecidos para los microorganismos patógenos, los productos afectados deberán ser excluidos del consumo humano. Las comidas preparadas no contendrán ningún tipo de microorganismo patógeno ni sus toxinas en una cantidad que afecta a la salud de los consumidores.

Rev Esp Salud Pública 2003, Vol. 77, N. ${ }^{\circ} 6$ 
El objetivo de este estudio es valorar la calidad microbiológica de las comidas servidas en los comedores de los colegios públicos de Tenerife, determinando si existen diferencias en los parámetros estudiados, entre los alimentos recogidos en los colegios donde se elabora la comida y en aquéllos servidos por catering y según los distintos tipos de platos y el tratamiento térmico al que hayan sido sometidos.

\section{MATERIAL Y MÉTODOS}

Se realizó un estudio epidemiológico descriptivo transversal, analizando 898 muestras de alimentos procedentes de comedores escolares de la isla de Tenerife. Mediante un muestreo aleatorio estratificado se seleccionó un total de 101 colegios públicos de la Isla de Tenerife, 58 con elaboración propia de comidas (gestión directa) y 43 con servicio de catering (gestión contratada). El tamaño total de la muestra se fijó en torno a 100 para obtener un margen de error máximo del 5\% en los datos globales. El tamaño muestral en cada estrato o sub-estrato se fijó por el tamaño proporcional a partir de la relación entre el tamaño poblacional y el tamaño de la muestra global $(\mathrm{ne} / \mathrm{Ne} \bullet \mathrm{n} / \mathrm{N})^{6}$.

Recogida de muestras y análisis microbiológico: Las muestras se recogieron en envases estériles, en las mismas condiciones en que se sirven a los escolares, debidamente etiquetados y cerrados. El transporte hasta el laboratorio se realizó en condiciones de refrigeración $\left(4-6^{\circ} \mathrm{C}\right)$ y se procedió lo antes posible a su análisis microbiológico. Se tomaron dos muestras por menú, una correspondiente al primer plato y otra al segundo. En algunos casos, se tomó asimismo una tercera muestra correspondiente a un complemento, aquellos alimentos que acompañan a un plato principal, y una cuarta correspondiente a una ensalada. En el estudio se analizaron un total de 898 muestras, 363 procedentes de colegios de gestión contratada y 535 de gestión directa. La distribución de las muestras analizadas según el tipo de gestión, plato y el tipo de tratamiento que llevan se observa en la tabla 1. En los primeros platos se incluyen principalmente cremas y purés de verduras, legumbres y sopas. El segundo plato está basado en arroz, pasta, huevos, pescado y carne. Entre los complementos incluimos salsas, patatas y hortalizas y las ensaladas, que en su totalidad son ensaladas mixtas.

Los parámetros estudiados han sido los recogidos en la Reglamentación vigente (RD 3484/2000, de 29 de diciembre) y las técnicas utilizadas han sido: aerobios mesófilos totales (ISO 4833), Enterobacteriaceae lactosa positiva (ISO 4831), Escherichia coli (ISO 7251), Staphylococcus aureus (ISO 6886), Salmonella (ISO 6579) y Listeria monocytogenes (ISO 11990-2) ${ }^{6}$.

Análisis estadístico: El análisis estadístico de la comparación de los resultados microbiológicos, según gestión de los comedores, platos analizados y tratamiento térmico, ha sido realizado con los programas Analysis y Statcalc del paquete informático Epi-Info V6.02 de los Centers for Disease Control (CDC) de Atlanta, EEUU, con un nivel de significación de $\mathrm{p}<0,01$ y $\mathrm{p}<0,05$. Con el programa Analysis se obtienen los listados, frecuencias y tablas de resultados, ordenándose los datos en forma individualizada o mediante cruce de variables. Con el programa Statcalc se realizó la prueba del chi cuadrado $\left(\chi^{2}\right)$, usando tablas de contingencia $2 \mathrm{xN}$ según cada caso, obteniéndose el valor de $\chi^{2}$ derivado de la fórmula corregida de Yates. Se dan los valores de $\mathrm{p}$ para 1 grado de libertad usando la fórmula de Poole y Borchers y si el valor de una de las celdillas fuese inferior a 5 se utiliza el cálculo exacto de probabilidades de Fisher según Rosner ${ }^{8}$.

\section{RESULTADOS}

En ninguna de las muestras analizadas se aislaron los patógenos Salmonella y Listeria 
Tabla 1

Distribución de las muestras analizadas según el tipo de gestión, plato y tratamiento

\begin{tabular}{|cccccc|}
\hline & \multicolumn{3}{c}{ A } & \multicolumn{3}{c|}{ B } & TOTAL \\
\cline { 2 - 5 } Muestras & C.n & C.C & C.D & C.C & \\
\hline I'iunci plats & $\ldots-$ & $\ldots$ & 180 & 128 & 313 \\
Ensaladas & 58 & 33 & $\ldots-$ & $\ldots$ & 91 \\
Scgundo plato & 11 & 4 & 206 & 150 & 371 \\
Complementos & 16 & 20 & 55 & 28 & 119 \\
TOTAI. & 76 & 62 & 459 & 301 & 898
\end{tabular}

GD: Gestión directa; GC: Gestión contratada; A: Comidas preparadas sin tratamiento térmico o con tratamiento térmico que lleven algún ingrediente no sometido a tratamiento térmico. B: Comidas preparadas con tratamiento térmico

monocytogenes. En las tablas 2, 3 y 4 se reflejan los valores de los recuentos obtenidos (por intervalos), en el total de comidas analizadas, para los microorganismos aerobios mesófilos totales, Enterobacteriaceae lactosa positiva o grupo coliformes totales y Escherichia coli, según el tipo de gestión, platos y tratamiento (Grupo A y B).

Los recuentos más elevados se obtuvieron para los microorganismos aerobios mesófilos totales. El 79\% de los alimentos analizados presentó recuentos para este parámetro, el $91 \%$ de las ensaladas y el $85 \%$ de los segundos platos. Para Enterobacteriaceae totales, el $15 \%$ de las muestras analizadas fueron positivas (89\% de ensaladas, el $11 \%$ de segundos platos y el $9 \%$ de los complementos). Escherichia coli se aisló en el $24 \%$ de las ensaladas, el $4 \%$ de los complementos y el $1 \%$ de los segundos platos y Staphylococcus aureus se aisló en tres alimentos todos ellos en comidas recogidas de colegios con comedores de gestión directa, 2 alimentos con ingredientes sin tratamiento térmico ( 1 ensaladilla y 1 salpicón de atún) y 1 alimento con tratamiento completo (pollo).
Al comparar las dos gestiones se observa como para aerobios mesófilos totales se obtiene un mayor porcentaje de muestras con recuentos positivos en la gestión contratada $(69,4 \%)$ que en gestión directa $(57 \%)$. Para E. coli el porcentaje de muestras positivas es similar en ambas gestiones $(14,6 \%)$ y para Enterobacteriaceae lactosa es de $17,1 \%$ en directa y $12,1 \%$ en contratada.

Si comparamos los alimentos que pertenecen al grupo A con los que corresponden al grupo $\mathrm{B}$, el número de muestras con recuentos positivos es superior en todos los parámetros estudiados, tanto en gestión directa como en gestión contratada, en los del grupo A ya que, como hemos indicado, incluye alimentos en los que todos o algunos de los ingredientes no han sido sometidos a tratamiento térmico. Así el porcentaje de muestras positivas para los distintos parámetros en los alimentos del grupo A fueron $86,2 \%$ para Aerobios mesófilos totales, $65,2 \%$ para Enterobacteriaceae totales y $15,9 \%$ para E. coli. En los del grupo B fueron $66,6 \%$ para Aerobios mesófilos totales, 2,5\% para Enterobacteriaceae totales y $1,3 \%$ para E. coli. En cuanto al tipo de plato,

Rev Esp Salud Pública 2003, Vol. 77, N. ${ }^{\circ} 6$ 
ESTUDIO MICROBIOLÓGICO DE LAS COMIDAS SERVIDAS EN LOS COMEDORES ESCOLARES DE LA ISLA DE TENERIFE

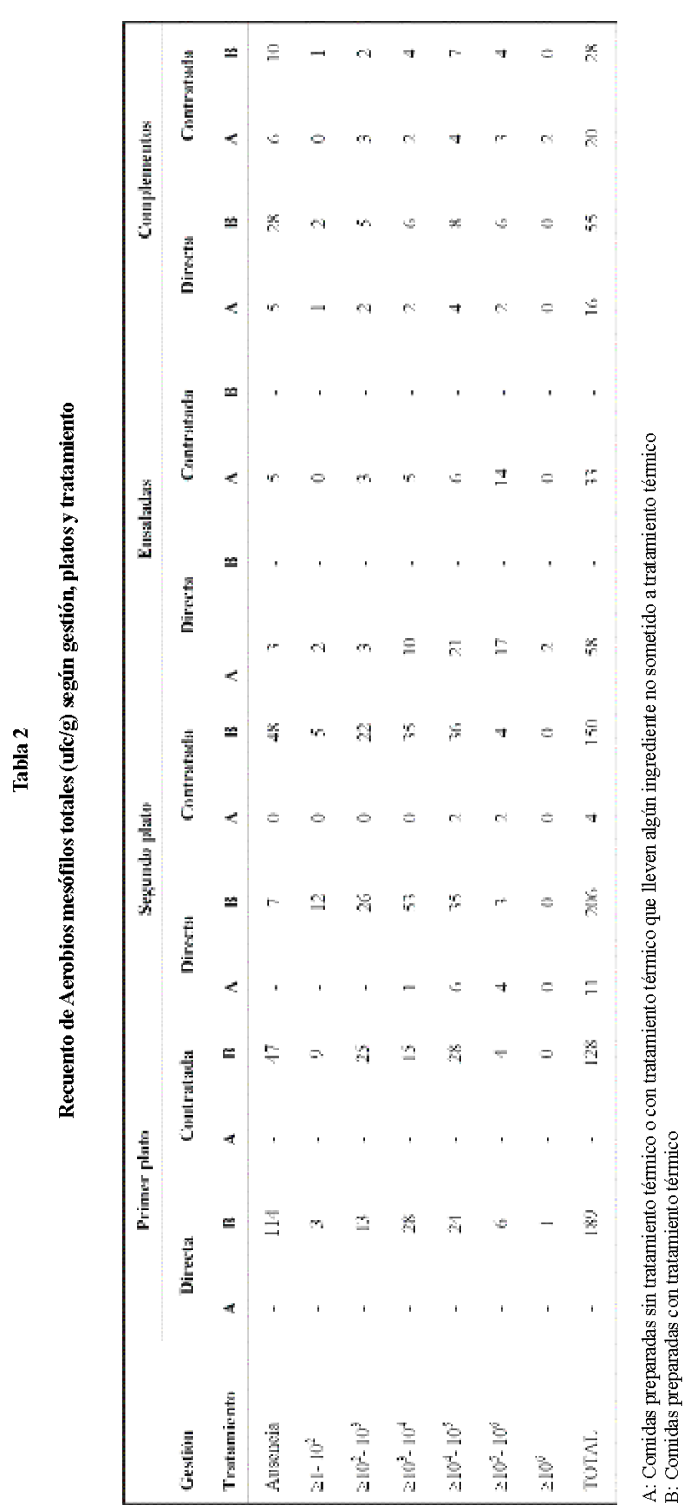

Rev Esp Salud Pública 2003, Vol. 77, N. ${ }^{\circ} 6$ 


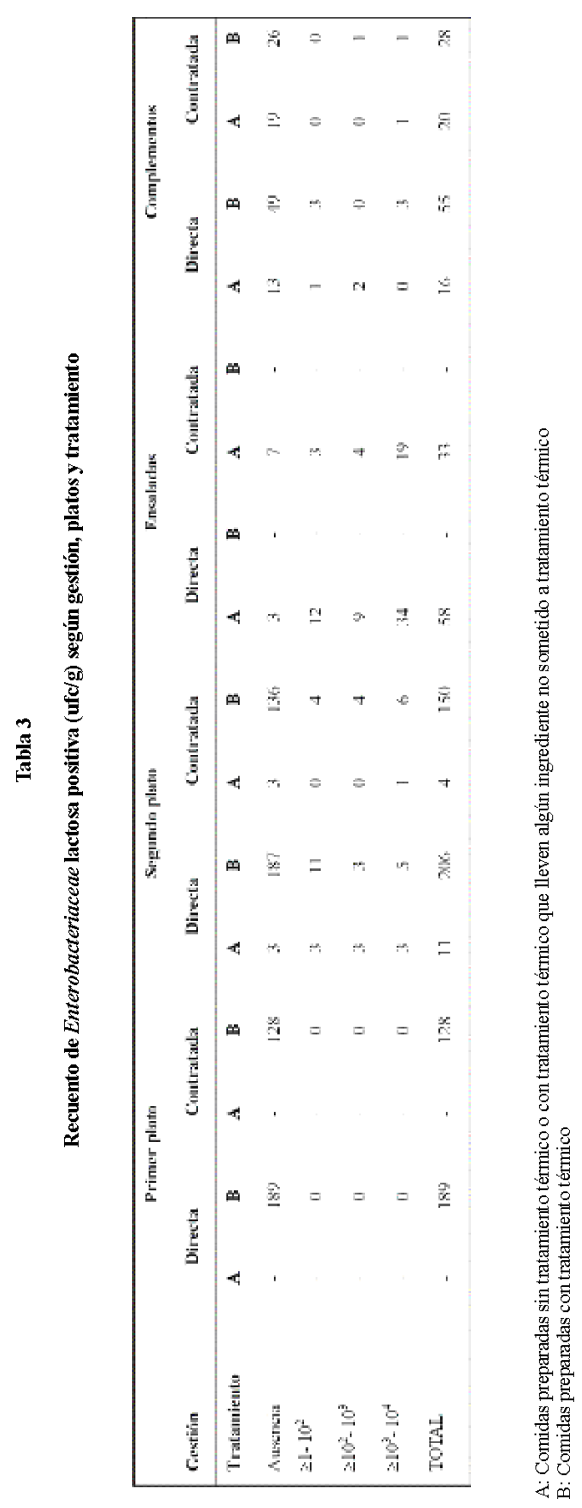


ESTUDIO MICROBIOLÓGICO DE LAS COMIDAS SERVIDAS EN LOS COMEDORES ESCOLARES DE LA ISLA DE TENERIFE

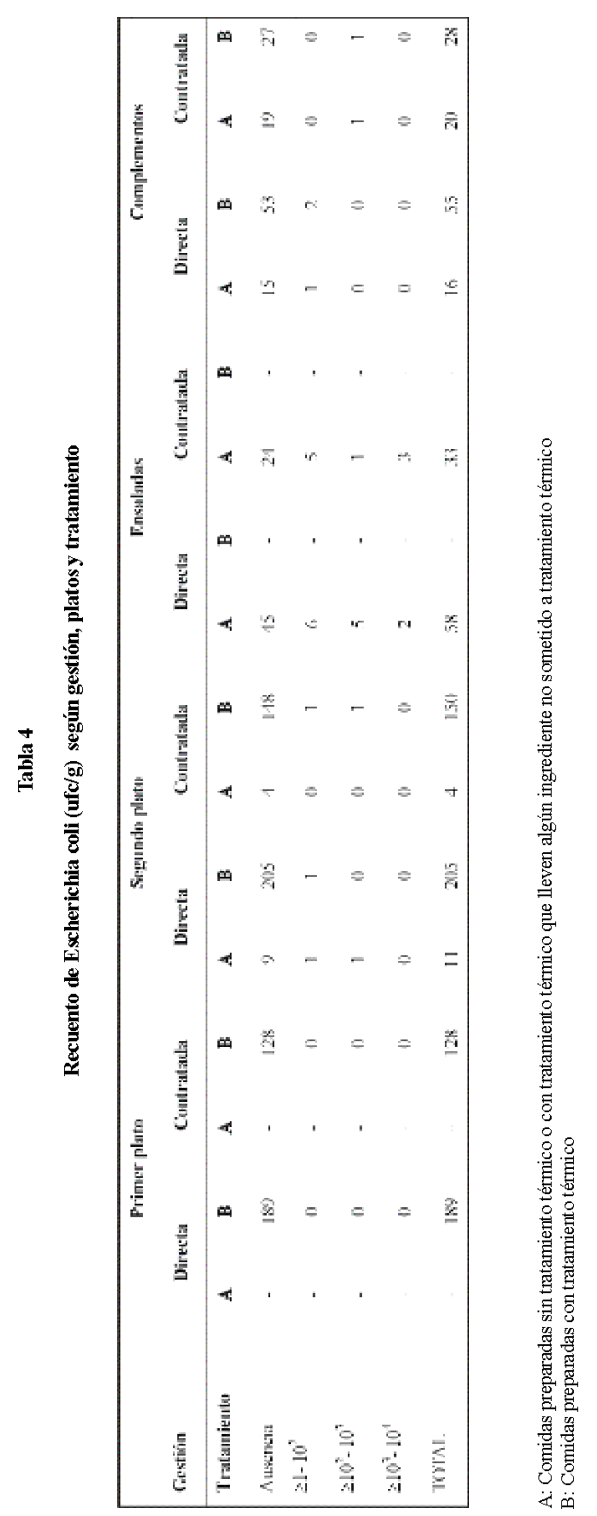

Rev Esp Salud Pública 2003, Vol. 77, N. ${ }^{\circ} 6$ 
y tal como se indica en las tablas 2, 3 y 4, son las ensaladas los alimentos que presentan los valores más elevados en los parámetros estudiados.

Con relación a los aerobios mesófilos totales, también se obtienen valores elevados de muestras positivas en los primeros y segundos platos y en los complementos, tanto en gestión directa como contratada. Para Enterobacteriaceae lactosa positiva, son las ensaladas las que presentan recuentos más elevados, seguido de los segundos platos y complementos.

Con relación a Escherichia coli, en gestión directa se detecta en 13 muestras de ensalada ( $22 \%$ del total), en 3 segundos platos $(19 \%)$ y en 3 complementos (4\%). En gestión contratada se detecta en 9 muestras de ensaladas (27\%), 2 muestras de segundos platos $(1 \%)$ y 2 complementos (4\%).

\section{DISCUSIÓN}

Del total de muestras analizadas un $8,24 \%$ de las mismas superan uno o más de los límites establecidos para los parámetros estudiados. No podemos comparar nuestros resultados con los mencionados por otros autores, ya que los trabajos consultados hacen referencia a legislaciones anteriores ya derogadas ${ }^{9,10}$. En ambas legislaciones los límites eran diferentes, más estrictos que los actuales, salvo en los patógenos.

Torre y cols. ${ }^{11}$ en el análisis de la calidad microbiológica de platos elaborados en establecimientos de restauración colectiva, encuentran que un $38,2 \%$ de las muestras presentan uno o varios parámetros microbiológicos superiores a lo normal, con elevados recuentos de aerobios mesófilos totales y enterobacterias.

En nuestro estudio, 29 muestras (3,2\% del total) superan los límites establecidos para aerobios mesófilos totales (tabla 5). El lími- te establecido en la legislación es de $10^{6}$ ufc/g en los alimentos del grupo A y $10^{5}$ ufc/g en los alimentos del grupo B. Destacan 11 primeros platos, todos ellos con tratamiento térmico, y 12 complementos, en su mayoría correspondientes al grupo B, lo que puede indicar que el tratamiento térmico ha sido insuficiente o bien que estos alimentos, una vez finalizado su proceso de cocinado y hasta el momento de ser servidos a los escolares, no se mantienen en las condiciones térmicas adecuadas. El porcentaje de muestras que superan los límites para aerobios mesófilos totales en gestión contratada $(3,85 \%)$ es algo superior que el encontrado para gestión directa $(3,36 \%)$, si bien esta diferencia no es estadísticamente significativa.

Los organismos mesófilos crecen en temperaturas óptimas de 30 y $45^{\circ} \mathrm{C}$, se les encuentra en alimentos almacenados a temperatura ambiente o en alimentos refrigerados cuando se ha roto la cadena del frío ${ }^{12}$. Recuentos altos en alimentos estables a menudo indican materias primas contaminadas o tratamientos no satisfactorios desde el punto de vista sanitario, mientras que en los productos perecederos pueden indicar también condiciones inadecuadas de duración y/o temperatura durante su almacenamiento. La presencia de un número elevado de bacterias aerobias mesófilas que crecen bien a temperatura corporal o próxima a ella, significa que pueden haberse dado condiciones favorables a la multiplicación de los microorganismos patógenos de origen humano o $\operatorname{animal}^{4,13}$.

En el estudio de Pérez-Silva ${ }^{14}$ realizado en 44 comedores escolares (90 muestras analizadas) se encuentra que un $48,7 \%$ de las muestras presentan recuentos superiores al límite establecido y Jurado y cols ${ }^{15}$ en un estudio realizado en comedores colectivos encuentra que un $22,7 \%$ de las muestras analizadas superan los límites para este parámetro, si bien hay que indicar que en estos estudios se toma el límite establecido por la

Rev Esp Salud Pública 2003, Vol. 77, N. ${ }^{\circ} 6$ 
ESTUDIO MICROBIOLÓGICO DE LAS COMIDAS SERVIDAS EN LOS COMEDORES ESCOLARES DE LA ISLA DE TENERIFE

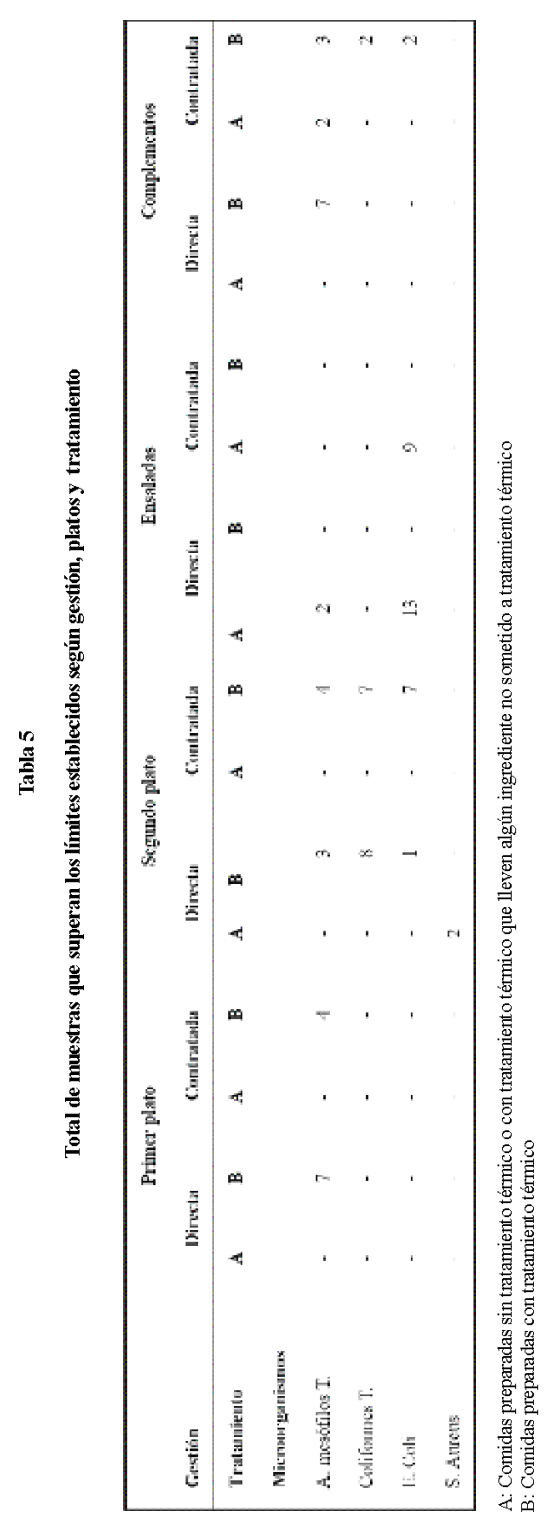

Rev Esp Salud Pública 2003, Vol. 77, N. ${ }^{\circ} 6$ 
legislación anterior $\left(10^{3} \mathrm{ufc} / \mathrm{g}\right.$ en comidas calientes y $10^{5} \mathrm{ufc} / \mathrm{g}$ en comidas frías).

Con relación a los coliformes totales, se sobrepasan los límites en 23 muestras $(2,6 \%)$, que en su mayoría (18) corresponden a segundos platos, todos ellos alimentos en los que todos sus ingredientes han sufrido un tratamiento completo en su cocinado. La presencia de niveles considerables de Enterobacteriaceae lactosa positiva o de coliformes indica un tratamiento inadecuado y/o contaminación posterior al tratamiento, más frecuentemente a partir de materias primas, equipos sucios o manejo no higiénico. Esta multiplicación microbiana pudiera haber permitido el crecimiento de toda la serie de microorganismos patógenos y toxigénicos ${ }^{13}$. Para este grupo el porcentaje de muestras que superan los límites es mayor en gestión contratada $(3,3 \%)$ que en gestión directa $(2,1 \%)$, aunque la diferencia no es significativa.

En nuestro estudio, para Escherichia coli, se ha sobrepasado el límite $\left(\leq 10^{2} \mathrm{ufc} / \mathrm{g}\right)$ en un $2,2 \%$ del total de las muestras, en 4 segundos platos, 4 complementos y en 11 ensaladas. Si consideramos la gestión, los porcentajes son similares.

La presencia de E. coli en un alimento no constituye un indicador directo de la presencia de un patógeno sino que implica únicamente un cierto riesgo de que pudiera estar presente. Al ser un huésped constante del intestino del hombre y de los animales de sangre caliente, se considera como un buen indicador de contaminación fecal. Tiene el inconveniente de vivir poco tiempo fuera del intestino, por lo que su presencia en los alimentos indica contaminación fecal reciente. Recuentos elevados de este microorganismo en un alimento sugieren una falta general de limpieza en el manejo del mismo y un almacenamiento inadecuado ${ }^{4,16,17}$. Se destruye con facilidad a temperaturas de pasteurización y mediante la apropiada cocción de los alimentos, así como durante su almacenamiento en frío.
La presencia de Staphylococcus aureus, al igual que la de E. coli, se considera como testigo de falta de higiene. Se multiplica rápidamente a temperatura ambiente y si existe un tratamiento térmico insuficiente puede desarrollarse con mayor facilidad. Principalmente su contaminación procede de vías orales, nasales, piel, entre otros. Además, el material y equipos sucios y las materias primas de origen animal pueden ser asimismo la fuente de la contaminación. Es una especie muy sensible a la acción del calor y de los desinfectantes. Su presencia o la de sus toxinas es signo evidente de falta de higiene $\mathrm{e}^{4,16,17}$.

En nuestro estudio se obtiene que un 2,35\% de muestras del grupo A de gestión directa sobrepasan el límite de este parámetro $\left(\leq 10^{2} \mathrm{ufc} / \mathrm{g}\right)$ mientras que en comidas procedentes de comedores de gestión contratada no se aísla este microorganismo. En el estudio ya mencionado de Jurado-Pérez y cols ${ }^{15}$ un $5,62 \%$ de las muestras presentan recuentos superiores al límite establecido para este microorganismo.

Si comparamos el total de platos que superan los límites según el tipo de gestión, se observa que el porcentaje en gestión directa es de $7,5 \%$ y en gestión contratada del $9,4 \%$, si bien en el análisis estadístico estas diferencias no son significativas.

En gestión directa al analizar el porcentaje de muestras que superan los límites, según el tipo de plato, se observa como las ensaladas $(15,5 \%)$ y los complementos $(15,5 \%)$ son los que presentan mayores incumplimientos. No se observan diferencias significativas entre ellos. Se observan diferencias significativas $(\mathrm{p}<0,01)$ entre los primeros platos $(3,7 \%)$ y las ensaladas y entre los primeros platos y segundos platos (sobrepasan los límites un 6,0\%) con los complementos. Existe un porcentaje muy bajo de muestras que superan los límites de primeros platos (siempre para aerobios mesófilos totales), lo que pone de manifiesto que el tratamiento

Rev Esp Salud Pública 2003, Vol. 77, N ․ㅜ 6 
térmico y su posterior almacenamiento al que han sido sometidos fue el adecuado. Hay diferencias significativas $(\mathrm{p}<0,05)$ entre los segundos platos y las ensaladas y entre los primeros y los segundos platos.

En gestión contratada, al analizar el porcentaje de muestras no aptas según el tipo de plato, se observa como son las complementos $(20,8 \%$ de no aptos) y las ensaladas (12,1\% de no aptas), los que presentan mayores incumplimientos. No se observan diferencias significativas entre ellos. Se observan diferencias significativas $(\mathrm{p}<0,05)$ entre los primeros platos $(3,1 \%$ no aptos) con las ensaladas y los segundos platos y entre los primeros platos y complementos $(\mathrm{p}<0,01)$.

Según los distintos platos, si comparamos por gestión, no existen diferencias significativas, para ninguno de ellos.

Con relación a las ensaladas, en un estudio de Riba-Sicart ${ }^{18}$ refleja que al aplicar un plan de mejora en la elaboración de las ensaladas en los comedores universitarios, sobre todo con un control periódico del cumplimiento de la utilización de hipoclorito sódico en el lavado de hortalizas crudas, el porcentaje de muestras no aptas disminuye de forma importante.

Si analizamos el número de muestras no aptas diferenciando por tratamiento (alimentos del grupo A ó B), se observan diferencias significativas en el total de muestras analizadas, y según tipo de gestión.

La legislación vigente diferencia, como ya hemos mencionado, en alimentos del grupo A, si alguno o todos los ingredientes han sido sometidos a tratamiento culinario y alimentos del grupo B, con todos los ingredientes con tratamiento culinario. Los límites de los indicadores y Escherichia coli son diferentes según el tipo de tratamiento, siendo más permisivo en los alimentos del grupo $\mathrm{A}$. Por ello, como se observa en las tablas 2, 3 y
4 el número de alimentos del grupo A con valores de recuento de los parámetros mayores, al aplicar el límite microbiológico los alimentos del grupo A sobrepasan los límites un $12 \%$ y los del grupo B un 7,5\%, y como además el número de alimentos analizados del Grupo B es muy superior a los del grupo A, las diferencias entre ellos son significativas.

En conclusión, podemos afirmar que la calidad microbiológica de las comidas servidas en los comedores escolares de la isla de Tenerife es aceptable, si bien existe un porcentaje de alimentos que superan los límites establecidos para los microorganismos indicadores y en testigos de falta de higiene. Siendo los comedores escolares utilizados por un colectivo muy vulnerable, se deben revisar los métodos de vigilancia aplicados en los puntos de control crítico.

\section{BIBLIOGRAFÍA}

1. Aranceta J. Nutrición en la edad evolutiva. En: Serra L, Aranceta J, Mataix J. Nutrición y Salud Pública. Métodos, bases científicas y aplicaciones. Barcelona: Editorial Masson; 1995; p. 185 92.

2. Aranceta J, Pérez-Rodrigo C. Consumo de alimentos y estado nutricional de la población escolar de Bilbao. Bilbao: Área de Salud y Consumo del Ayuntamiento de Bilbao;1996.

3. Arias C, Blanco N, Rodríguez A, Tardón A, Cueto A. Condiciones higiénico-sanitarias de comedores escolares del municipio de Oviedo. Rev Esp Salud Pública 1998; 72: 571-81.

4. Pascual Anderson MR, Calderón y Pascual V. Microbiología alimentaria. Metodología analítica para alimentos y bebidas. Madrid: Díaz de Santos; 1999 .

5. Boletín Oficial del Estado. Real Decreto $3484 / 2000$, de 29 de diciembre, por el que se establecen las normas de higiene para la blecen las normas de higiene para la elaboración, distribución y comercio de comidas preparadas.

6. Scheaffer RL, Mendenhall W, Ott L. Elementos de muestreo. México: Iberoamericana; 1987. p. 58-9. 
7. Allaert C, Escolá M. Métodos de análisis microbiológicos de los alimentos. Madrid: Díaz de Santos; 2002

8. Centers for Disease Control. Epi-Info V6.02. Atlanta: Centers for Disease Control; 2000

9. Boletín Oficial del Estado. Orden de 21 de febrero sobre Normas higiénico-sanitarias para la instalación y funcionamiento de industrias dedicadas a la ción y funcionamiento de industrias dedicadas a la
preparación y distribución de comidas para consupreparación y distribución de comidas para consu-
mo en colectividades y medios de transporte. BOE mo en colectivi
$59,10 / 3 / 77$

10. Boletín Oficial del Estado. RD 2817/1983, de 13 octubre, por el que se aprueba la Reglamentación Técnico-Sanitaria de los Comedores Colectivos. BOE núm 270 de 11/11/1983.

11. Torre R, Jurado R, Pérez J, Hernández M, Morales E, Jurado M. Calidad microbiológica de platos elaborados en establecimientos de restauración. Rev Esp Nutr Comunitaria 1998; 4: 293-4.

12. Bourgeois CM, Mescle JF, Zucca J. Microbiología alimentaria. Vol I. Zaragoza: Acribia; 1994.

13. The International Commission on Microbiological Specifications for Foods (ICMSF). Microorganis- mos de los alimentos 1 . Su significado y métodos de enumeración. $2^{\mathrm{a}}$ ed. Zaragoza: Acribia;, 2000.

14. Pérez-Silva MC, Belmonte S, Martínez J. Estudio microbiológico de los alimentos elaborados en comedores colectivos de alto riesgo. Rev Esp Salud Pública 1998; 72: 67-75.

15. Jurado-Pérez R, Pérez-Aparicio J, De la Torre R, Alonso R, Martínez A, Morales E, HernándezBienes M. Calidad microbiológica de platos en comedores colectivos. Aliment 1999; Julio-Agosto: $35-40$

16. Bell C, Alec Kyriakides. E. coli. Una aproximación práctica al microorganismo y su control en los alipráctica al microorganismo y su
mentos. Zaragoza: Acribia; 2000.

17. Smoot LM, Pierson MD. Microorganismos indicadores y criterios microbiológicos. En: Microbiología de los Alimentos. Fundamentos y fronteras. Doyle MP, Beuchat LR, Montville TJ. Eds. Zaragoza: Acribia; 2000.p. 69-83.

18. Riba-Sicart M, Roig-Sagués A, Hernández-Herrero MM, Rodríguez-Jerez JJ, Mora-Ventura MT. Calidad higiénico-sanitaria de las ensaladas servidas en comedores universtarios. Aliment 1998; Noviembre: 63-5. 